\title{
Web bounty hunters chase research patients
}

A Massachusetts dot-com has taken on the role of Indiana Jones in the jungles of high-tech patent law. BountyQuest, a new Internet site that trades hard-to-find intellectual property information for quick rewards has set up an online "most wanted" board that has attracted a worldwide network of sleuths by offering bounties in exchange for damaging dirt on biotech and software patents.

Since its launch last fall, BountyQuest has posted several dozen rewards for 'prior art', as it is known, on patented biotech inventions, ranging from pharmaceutical treatments for erectile dysfunction to a method for the overexpression of human $\alpha$-galactosidase A in mammalian cells. Many of the information seekers are looking to avoid licensing fees or shoot down a patent infringement case, and don't want to pay the tens or hundreds of thousands of dollars that a good attorney might charge for a prior art search.

The latest target is California-based Incyte Genomics, whose relational data-

\section{FDA tightens human oversight}

As part of a continuing overhaul of systems designed to protect human research subjects in the United States, the Food and Drug Administration (FDA) has established the Office for Human Research Trials (OHRT), which will coordinate the agency's efforts to monitor clinical trials of new therapies and devices.

The creation of the new FDA office parallels a step taken in September by the Department of Health and Human Services, which replaced the National Institutes of Health's Office for Protection from Research Risks with a higher-level office called the Office for Human Research Protection (OHRP). While the OHRP oversees basic clinical research, the OHRT will monitor industry-sponsored research aimed at getting new drugs and devices approved. The two offices are supposed to coordinate their efforts in order to maintain consistent regulations for both types of research.

In addition to addressing increasing public fears about clinical trial safety, the new office could help streamline base patent was undermined by BountyQuest last month. In response to an anonymous offer of a $\$ 10,000$ bounty, German graduate student Holger Blasum tracked down evidence that seems to invalidate one of Incyte's 10 genomic database patents. The patent was filed in 1997, but Blasum, a computer scientist with a background in molecular biology, realized that most genomic database development projects in the mid-1990s were collaborative.

After a quick literature search, Blasum found two journal articles on the same subjects from 1993 and 1994, and submitted them to the website. "It seemed easy enough to do, and because I have some understanding of the patent system I know there are lots of bogus patents," says Blasum. "This was obviously one of the very weak ones."

According to BountyQuest's patent attorney, this evidence of prior art poses as a serious challenge to Incyte's proprietary claim on the technology. But Incyte general counsel Lee Bendekgey says that no legal tussles or licensing agreements are currently pending with regard to the database in question, and as a result, Blasum's discovery will not have any immediate impact. "We still don't know what this means for the patent in question," says Bendekgey. "We may end up sending it back to the [United States] Patent and Trademark Office and asking them to re-examine the patent to see if they think we are still entitled to it. We don't want to be a company that people think of as having or trying to get invalid patents, so if this invalidates our patent I would just as soon know about it."

Either way, Blasum gets $\$ 10,000$ for his work, a quarter of which he intends to donate to a European anti-patent organization. And there are apparently no hard feelings on Incyte's part. In fact, Bendekgey says he wouldn't rule out using the service himself. "For $\$ 10,000$, you can have the whole world looking for prior art for you," helaughs. "It's a pretty cheap way to find out."

Kathleen McGowan, New York

\section{Biologist becomes first woman to lead Princeton}

Princeton University has appointed Shirley Tilghman as the first female president in its 254-year history. Tilghman, a molecular biologist, has been a professor at Princeton for 15 years and will become only the second ever woman president of an Ivy League University when she takes office on 15 June. Judith Rodin, a Professor of Psychology, Medicine and Psychiatry, was inaugurated as president of the University of Pennsylvania in 1994 and Ruth J. Simmons will become president at Brown University, on 1 July.

Tilghman, who has headed Princeton's Institute for Integrative Genomics for the past two years, recently appeared before Congress to argue on behalf of continued funding for stem cell research despite the vocal objections of pro-life suporters. A lifelong advocate of female leadership within the scientific community, she obtained her Ph.D. in biochemistry from Temple University and embarked on a research career studying mammalian embryonic development at the US National Institutes of Health.

\section{Stephen Horwitz, New York}

FDA oversight. "It brings together the issues about human research protection much more clearly across the three [FDA] centers that deal with drugs, devices and biologics," says Roger Meyer, senior consultant in clinical research for the Association of American Medical Colleges (AAMC; Washington, DC). Meyer explains that previously, each center within the FDA was responsible for monitoring trials within its jurisdiction, making the bureaucratic requirements for clinical researchers more complex.

David LePay, director of the OHRT, expects the department to be fully functional by the end of the year. In the interim period, a new Web site explaining FDA clinical trial regulations serves as a point of contact for researchers (http://www.fda.gov). 